\section{Heros de la promotion de la sante}

La Revue canadienne de médecine interne générale - votre revue - existe pour promouvoir l'érudition en médecine interne générale et pour sensibiliser les gens aux questions importantes dans nos pratiques. J'ai le plaisir d'annoncer dans la Revue une nouvelle initiative visant à diffuser les travaux du Comité de promotion de la santé du CSMI. Ce groupe est dirigé avec compétence (et enthousiasme !) par le Dr Bert Govig et vise à appuyer les activités de promotion de la santé des membres du CSMI et d'ailleurs. Chaque numéro présentera l'activité d'un héros de la promotion de la santé (HP). Le premier à être reconnu dans notre prochain numéro du CJGIM est le Dr Thomas Brothers, qui a également reçu la bourse d'études Hui Lee en promotion de la santé.

Il est important de se concentrer à nouveau sur la promotion de la santé parce que beaucoup d'entre nous ont été attirés par la médecine pour prévenir la maladie et la souffrance. Cependant, notre formation et la structure du système de soins de santé concentrent notre attention sur les défauts biologiques mesurables, ce qui nous amène, pour la plupart d'entre nous, à demeurer occupés à diagnostiquer et à traiter les patients atteints de problèmes aigus et chroniques. Malgré les réalités de notre pratique quotidienne, nous devons trouver le temps (même s'il y en a peu) de chercher un îlot de promotion de la santé tout en naviguant dans la mer du diagnostic, du traitement et de la gestion de crise à court terme.
La promotion de la santé peut avoir plusieurs visages. Il y a vingt ans, j'ai participé à l'élaboration de lignes directrices fondées sur des données probantes pour la prévention et le traitement de l'obésité. À l'époque, nous avons trouvé peu de données probantes pour guider les cliniciens vers des programmes de prévention communautaires efficaces ou vers des stratégies de prévention ou de traitement pour les individus. C'était frustrant, car notre intuition était que les interventions axées sur la population, comme l'éducation physique obligatoire de la 1re à la 12e année, avaient un sens. Comme nous le savons, le manque de preuves n'est pas la même chose que le manque d'effet, et dans ce cas-ci, c'est peut-être dû à un manque de financement de la recherche. La promotion de la santé est maintenant une priorité pour les gouvernements et les systèmes de soins de santé partout dans le monde, et les nouvelles recherches commencent à aborder une gamme croissante d'interventions portant sur les déterminants sociaux de la santé. Nous sommes enthousiastes à l'idée de rehausser le profil de la promotion de la santé grâce à cette nouvelle rubrique du Journal et nous avons hâte d'être inspirés et éduqués par nos héros HP.
James Douketis, MD

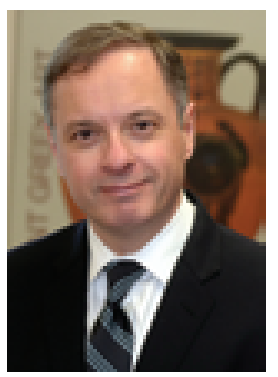

Éditeur en chef 